\title{
Radio-frequency probes of Antarctic ice at South Pole
}

\author{
D. Besson ${ }^{1}$ and I. Kravchenko ${ }^{2}$ \\ ${ }^{1}$ University of Kansas, Department of Physics and Astronomy, Lawrence, KS 66045-7582, USA \\ ${ }^{2}$ University of Nebraska - Lincoln, Department of Physics and Astronomy, Lincoln, NE 68588-0299, USA \\ Correspondence to: D. Besson (zedlam@ku.edu)
}

Received: 1 October 2012 - Published in The Cryosphere Discuss.: 14 November 2012

Revised: 25 March 2013 - Accepted: 3 April 2013 - Published: 16 May 2013

\begin{abstract}
Using hardware developed for the ARA (Askaryan Radio Array) particle astrophysics experiment, we herein report on the amplitude and temporal characteristics of polarized surface radar echo data collected in South Polar ice using radio sounding equipment with 0.5 -ns echo-time sampling. We observe strong echoes at $6,9.6,13.9,17$, and $19 \mu \mathrm{s}$ following vertical pulse emission from the surface, corresponding to reflectors in the upper half of the ice sheet. The synchronicity of those echoes for all broadcast azimuthal polarizations affirms the lack of observable birefringence over the upper half of the ice sheet. Of the five strongest echoes, three exhibit an evident amplitude correlation with the local surface ice flow direction, qualitatively consistent with measurements in East Antarctica. Combined with other radio echo sounding data, we conclude that observed birefringent asymmetries at South Pole are generated entirely in the lower half of the ice sheet. By contrast, birefringent asymmetries are observed at shallow depths in East Antarctica.
\end{abstract}

\section{Introduction}

The general study of the interior structure of the polar ice sheets, both vis-a-vis impurities as well as the socalled crystal orientation fabric (COF) has been a significant sub-discipline of radioglaciology for the past half-century (Kamb, 1959; Azuma and Higashi, 1985; Schmid and Casey, 1986; Jacka and Budd, 1989; Van der Veen and Whillans, 1994; Siegert and Kwok, 2000; Matsuoka et al., 2003; Eisen et al., 2007; Matsuoka et al., 2012). In the current picture, radio frequency scattering is primarily the result of three different mechanisms as one probes from the ice surface down to the bed. In the uppermost portion of the ice sheet, scattering is dominated by layered density variations followed by
COF scattering due to changes in the crystal orientation in the deeper ice, presumably resulting from a combination of considerable hydrostatic pressure and the resistance to motion presented by the underlying bedrock. So-called "acid", or "conductivity" scattering (sulfates and nitrate layers, e.g., often associated with volcanic activity) permeates the entire thickness of ice. Near the bed is an "echo-free zone", in which the reflection strengths are typically smaller than current instrumental sensitivity (Drews et al., 2009). The three scattering types differ in the magnitude of the radar echoes they produce, as well as the frequency dependence of those radar echoes (Fujita et al., 1999).

With $\epsilon$ the complex dielectric permittivity, $f$ the frequency of radar being employed, $\sigma$ the conductivity of an acid layer and $\Delta$ the difference in either $\epsilon$ or $\sigma$ across some boundary, the magnitude of density (or acid) reflected power typically varies as the square of those differences, i.e., $\Delta_{\epsilon}^{2}$ (or $\Delta_{\sigma}^{2} / f^{2}$ ) (Paren, 1981). Since both $\Delta_{\epsilon}$ and $\Delta_{\sigma}$ are approximately independent of frequency in the $\sim \mathrm{MHz}$ radio frequency regime, we expect density scattering to be independent of frequency, and conductivity scattering to vary inversely with frequency, with some temperature dependence, as well. Whereas acid scattering may occur at any depth within the ice sheet, density contrasts tend to diminish with pressure, and should be largely unobservable below $r \sim 1 \mathrm{~km}$ depth (Fujita et al., 2000). In contrast to typically "thin" conductive and/or density contrast layers which are associated with "isochrones" (i.e, signals with timescales smaller than the experimental resolution) and therefore have signal durations that are intrinsically sub-ns scale, ice core analysis indicates that reorientation of the crystal fabric, and therefore COF scattering, typically occurs over tens of meters of ice thickness (Siegert and Kwok, 2000), corresponding to hundreds of nanoseconds in echo duration. By comparison, grain 
size changes can occur on much shorter sub-meter length scales.

Additionally, continuous Rayleigh scattering in the ice volume - due, e.g., to air bubbles trapped in the upper, unenclathrated ice with size much smaller than typical radio wavelengths - results in weak radar returns with an amplitude $A$ dependence on wavelength $\lambda$ as $A \propto 1 / \lambda^{4}$, and dependence on distance $r$ as $A \propto 1 / \lambda^{4}$. This volume scattering sets the baseline above which stronger returns from planar reflections ( $A \propto 1 / r$, for a radio transparent medium) may be visible. For the case where the scattering size of the planar reflectors is much greater than one wavelength, phase coherence across the scatterers is lost, and the amplitude decreases with distance $r$ as $1 / r^{2}$.

\subsection{Birefringence}

The presence of a preferred crystal orientation fabric can lead to an asymmetry in the radio wavespeed with azimuthal polarization; i.e., birefringence. Physically, this corresponds to a difference $\delta$ in the real permittivity $\epsilon^{\prime}$ for propagation along the extraordinary- vs. ordinary axes, normalized to the average real permittivity $<\epsilon^{\prime}>: \delta_{\epsilon^{\prime}} \equiv\left(\epsilon_{e}^{\prime}-\epsilon_{o}^{\prime}\right) /<\epsilon^{\prime}>$. In the simplest picture of two-dimensional ice crystals stacking in the horizontal plane, with the perpendicular ( $\hat{c}$-axis) correspondingly aligned with the vertical $\hat{z}$-axis, one would expect radio wave propagation along the vertical from the surface to the bedrock (i.e., with the electric-field polarization axis in the horizontal) to be independent of azimuth, and birefringence to be noticeable as a difference between horizontal vs. vertical signal propagation.

The local horizontal bulk ice motion, perhaps resulting from convergent/divergent ice flow over hundred-km length scales, produces an azimuthal asymmetry in the horizontal plane, and can give rise to an elliptical distribution of the single pole ice fabric. The ice flow direction and the local strain history thus suggest a "natural" axis which may result in a wavespeed asymmetry, as explored elsewhere (Wang et al., 2002). South Pole, being neither a dome nor an ice divide, represents an "intermediate" locale to study ice fabrics.

Although several positive observations of ice birefringence have now been made (Table 1), data on exactly at what depths birefringence is physically generated within the ice sheet is still incomplete. Data indicating correlations of birefringent effects with local ice flow are also not entirely concordant; at least one measurement found a lack of alignment between the implied birefringent basis and the surface ice flow direction (Doake, 1982; Woodruff and Doake, 1979).

\section{Measured azimuthal variation of radar echoes}

\subsection{Setup}

The Martin A. Pomerantz Observatory (MAPO) building, located within $1 \mathrm{~km}$ of the geographic South Pole, houses the

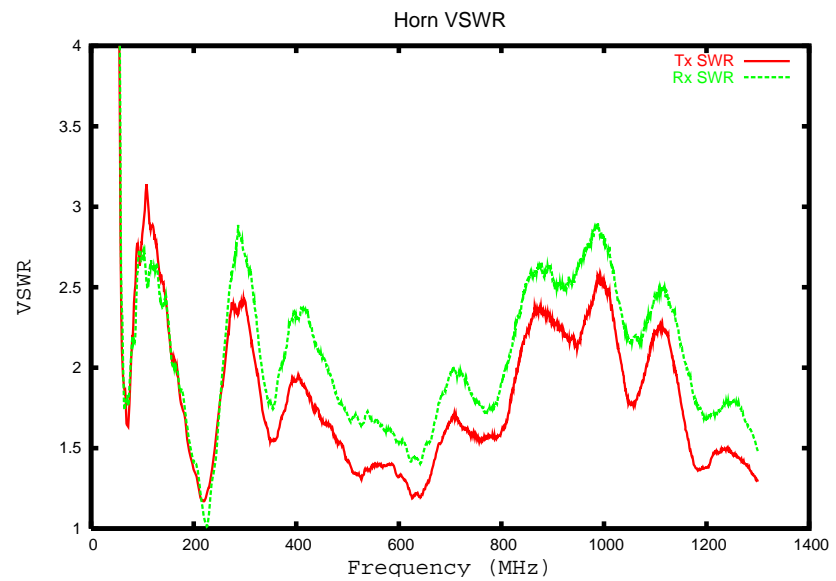

Fig. 1. Voltage Standing Wave Ratio (VSWR) for both transmitter $(\mathrm{Tx})$ and receiver $(\mathrm{Rx})$ horn used for data taken at South Pole.

signal generator and data acquisition system used for these measurements. Two $1.25 \mathrm{~cm}$ thick LMR-500 coaxial cables, each approximately $25 \mathrm{~m}$ long, were fed from within MAPO through a conduit at the bottom of the building and out onto the snow.

Each cable was then connected to a transverse electromagnetic (TEM) horn antenna on the snow surface (one for transmission and one for reception); these horn antennas are capable of transmitting or receiving linearly polarized signals. Constructed at the Institute of Nuclear Research in Moscow, these antennas were also used in our previous measurement of the ice attenuation length at the South Pole (Barwick et al., 2005). These antennas have good transmission characteristics, from $60 \mathrm{MHz}$ up to $1300 \mathrm{MHz}$, as indicated by their Voltage Standing Wave Ratio (VSWR) specifications. The VSWR represents a measure of the fraction of power delivered at the input port of an antenna which is broadcast into the environment, with a value of 1.0 representing $100 \%$ power transmission efficiency, and a value of 3.0 corresponding to $75 \%$ power transmission efficiency. The VSWR data for the two horn antennas used in this measurement are displayed in Fig. 1.

As with our previous measurement, for in-ice transmission, each horn antenna is placed facedown on the surface looking into the snow. We note that the measured frequency response shown in Fig. 1 corresponds to the horn antennas in their experimental configuration and therefore should correctly represent the horn characteristics relevant to this measurement. The forward gain of the horns is approximately $10 \times$ that of an isotropic emitter (i.e., $\sim 10 \mathrm{dBi}$ ) in air, or $\sim 12-15 \mathrm{dBi}$ in-ice. The co-polarization to cross-polarization isolation ("polarization purity") was measured at South Pole, and also in the lab at the University of Kansas, to exceed $10 \mathrm{~dB}$.

Signals were taken from an Avtech model AVIR-c fast pulse generator inside MAPO via coaxial cable out to the 
Table 1. Summary of recent birefringence measurements. Presented values of $\delta_{\epsilon^{\prime}}$ give the asymmetry between the "ordinary" vs. "extraordinary" measured real permittivities, corresponding to "fast" vs. "slow" in-ice wavespeeds. Fractional values (in percent) are normalized to the average ice dielectric permittivity at radio frequencies.

\begin{tabular}{llll}
\hline Group & Locale & $\delta_{\epsilon^{\prime}}$ Result & Comment \\
\hline (Hargreaves, 1977) & Greenland & $(0.024-0.031) \%$ & \\
(Woodruff and Doake, 1979) & Bach Ice Shelf & $0.52 \%$ & \\
(Fujita et al., 1996) & Alaska single crystals & $(1.2 \pm 0.2) \%$ & $9.7 \mathrm{GHz}$ \\
(Matsuoka et al., 1997) & Alaska single crystals & $\sim 1.1 \%$ & $1 \mathrm{MHz}-39 \mathrm{GHz}$ \\
(Matsuoka et al., 1998) & Dome Fuji core & $(0.33 \pm 0.0 .01) \%$ & $33 \mathrm{GHz}$ \\
(Doake et al., 2002) & Brunt Ice Shelf & $(>0.14-0.47) \%$ & \\
(Doake et al., 2003) & George VI Ice Shelf & $(>0.05-0.15) \%$ & \\
(Fujita et al., 2003) & Mizuho & measurable & $60 \mathrm{MHz}$ and $179 \mathrm{MHz}$ \\
(Fujita et al., 2006) & Mizuho & $(0.5-1.2) \%$ & $60 \mathrm{MHz}$ and $179 \mathrm{MHz}$ \\
(Besson et al., 2008) & Taylor Dome & $0.24 \%$ & time-domain \\
(Kravchenko et al., 2010) & South Pole & $0.31 \%$ & time-domain \\
\hline
\end{tabular}

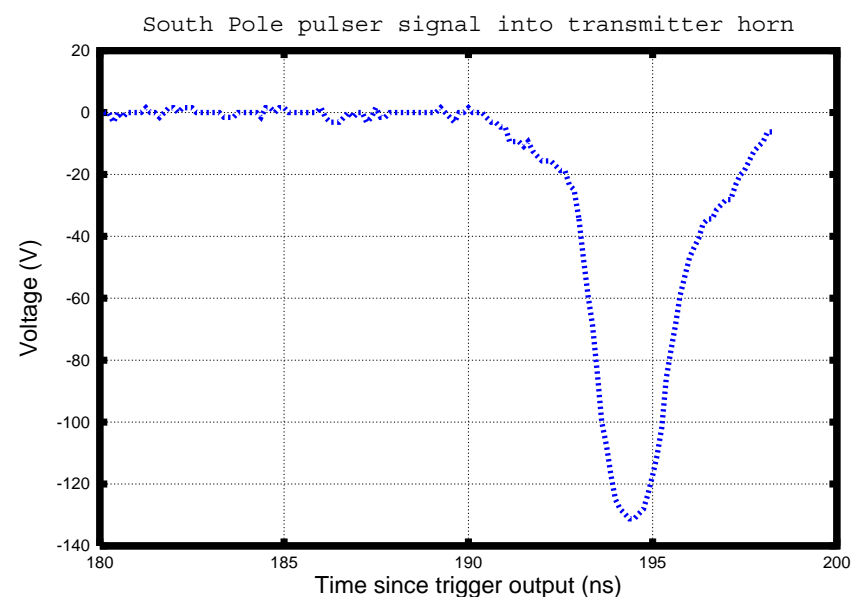

Fig. 2. Pulse generator output signal used for the primary measurements described herein. As seen in the figure, the total time delay through the associated cables has been measured to be 194 ns.

transmitter horn. Figure 2 illustrates the signal generator time domain voltage output for all of the pulses used for these measurements. During typical data-taking, the pulser is run at a repetition rate of $100 \mathrm{~Hz}$, although data accumulation is limited to $\sim 25 \mathrm{~Hz}$ by the throughput of our digital oscilloscope. At a distance of approximately $25 \mathrm{~m}$ from the transmitter horn, a separate coaxial cable connects the receiver horn antenna to the data acquisition electronics. Receiver horn signals are high- and low-pass filtered to remove components below $200 \mathrm{MHz}$ and above $1 \mathrm{GHz}$, notch filtered to suppress the large South Pole background noise at $450 \mathrm{MHz}$ which serves as the station Land Mobile Radio carrier, and finally amplified by $+52 \mathrm{~dB}$ prior to data acquisition and storage. This value of amplifier gain is sufficient to elucidate receiver signals above the intrinsic oscilloscope noise, but not so large that our receiver saturates on the large, initial through-air signals. For the low-noise
MITEQ amplifiers used in this measurement, the noise figure was reasonably low (1.8). Data acquisition of receiver horn waveforms was performed using a LeCroy 950 Waverunner digital oscilloscope. This scope features adequate bandwidth $(1 \mathrm{GHz})$ and a high maximum digitization speed (16 giga samples/sec [GSPS $\left.{ }^{-1}\right]$ ). For the measurements described herein, the scope sampling rate was generally set to $2 \mathrm{GSPS}^{-1}$. To enhance signal-to-noise, many waveforms (40 000 typically) were averaged. Trigger stability was ensured by splitting the output of the pulse generator, with one copy being sent to the transmitter horn antenna, and the other providing the trigger signal for the LeCroy scope.

We note three primary differences between our radio echo sounding (RES) apparatus, which was originally developed for application in Antarctica cosmic ray detection, and those typically employed for RES measurements:

1. We use a nanosecond-scale (unmodulated) transmitted pulse (Fig. 2), vs. "tone" signals of frequency 60-200 $\mathrm{MHz}$, having duration of order microseconds, providing considerably improved resolution of internal features.

2. Our receiver data acquisition samples at between $1 \mathrm{GSPS}^{-1}$ and $2 \mathrm{GSPS}^{-1}$, vs. sampling rates which are comparable to the monochromatic signal being broadcast. A high sampling rate is required in order to benefit from the use of short transmit pulses.

3. Reflections are reconstructed directly by real-time averaging, rather than synthesizing the reflected echogram image in post-processing. In principle, such averaging directly improves the signal-to-noise by a factor of $\sqrt{N}$, with $N$ the number of waveform captures (i.e., pulses) detected. 


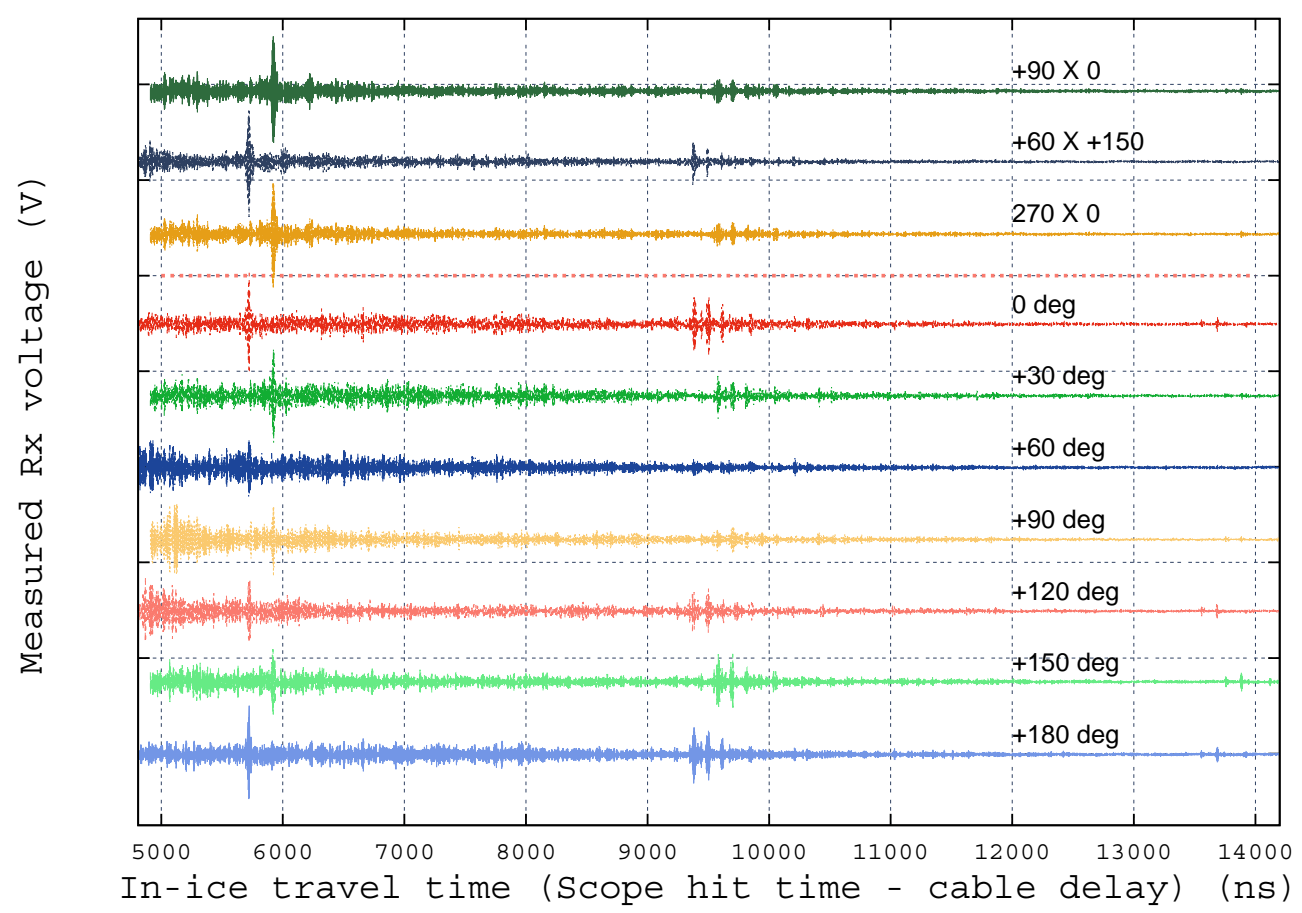

Fig. 3. Ensemble of echo amplitudes observed as a function of azimuthal orientation, for both co-polarized and cross-polarized broadcast signals, for echo times between $5 \mu \mathrm{s}$ and $14 \mu \mathrm{s}$. A DC offset has been added vertically to successive voltage traces for visual clarity; alternate waveforms are similarly also offset by $\pm 100 \mathrm{~ns}$ along horizontal. Each division vertically corresponds to $10 \mathrm{mV}$.

\subsection{Experimental results}

\subsubsection{Full azimuthal scan}

Figures 3 and 4 display the measured voltage as a function of echo time, as the polarization orientation of the transmitter and receiver is rotated. For ease of display, echograms have been vertically offset and alternately displaced by $\pm 100 \mathrm{~ns}$ relative to each other. In actuality, the most prominent echoes are observed to be synchronous within one nanosecond of each other, up to $19 \mu$ s echo time, and thus exclude the possibility of a birefringent effect in the upper half of the ice sheet, in contrast to the East Antarctica measurements (Fujita et al., 2003, 2006), for which birefringence was inferred at depths within $1 \mathrm{~km}$ of the surface. Although the birefringent asymmetry in East Antarctica, as measured interferometrically, was not entirely quantified, the information provided in those papers implies an expectation of $>10 \mathrm{~ns}$ temporal variation in observed return time as the transmitter and receiver sweep through the entire azimuth, inconsistent with our measurements at South Pole.

Antennas aligned with MAPO were assigned a zerodegree orientation; for reference, the surface ice flows in a direction corresponding to roughly $+153^{\circ}$ in these coordinates.

In these figures, for cross-polarized orientations, the notations " $\mathrm{A} \times \mathrm{B}$ " designate the azimuthal polarization orientation of transmitter ("A") and receiver ("B"), respectively:
"60 $\times 150$ " indicates a cross-polarization orientation with transmitter at $60^{\circ}$ and receiver at $150^{\circ}$; cross-polarization orientations are denoted by inclusion of the " $x$ ".

Two features are immediately evident from Figs. 3 and 4: (a) the presence of several short-duration returns at approximately 6, 9.6, 13.9, 17 and $19 \mu$ s with comparable shapes; and (b) for the $9.6 \mu$ s echo, we observe a sequence of three distinct echoes, separated by approximately $0.1 \mu \mathrm{s}$.

To display the waveforms in detail, Figs. 5, 6, and 7 show zooms of the reflections corresponding to the time intervals around $6 \mu \mathrm{s}, 9.6 \mu \mathrm{s}$ and $13.9 \mu \mathrm{s}$, illustrating the similarity of the reflection arrival time. We use these figures to also correlate our measurements with perhaps more familiar units of depth by translating reflection times to distances, using a temperature-weighted average EM wave velocity of $169 \pm 0.3 \mathrm{~m} / \mu$ s below the firn (Dowdeswell and Evans, 2004); through the firn, we use direct measurements of radio propagation wavespeed at South Pole (Kravchenko et al., 2005).

\subsection{Cross-polarized power}

For the shallowest reflection at $6 \mu$ s, the cross-polarized reflected power seems to exceed the power measured in the co-polarized orientation by an amount roughly four times larger than that allowed by the known cross-polarization isolation of our horn antennas. Reflection from a pure layer of incoherent scatterers would naively lead to equal power re-radiated isotropically, but will not necessarily change the 


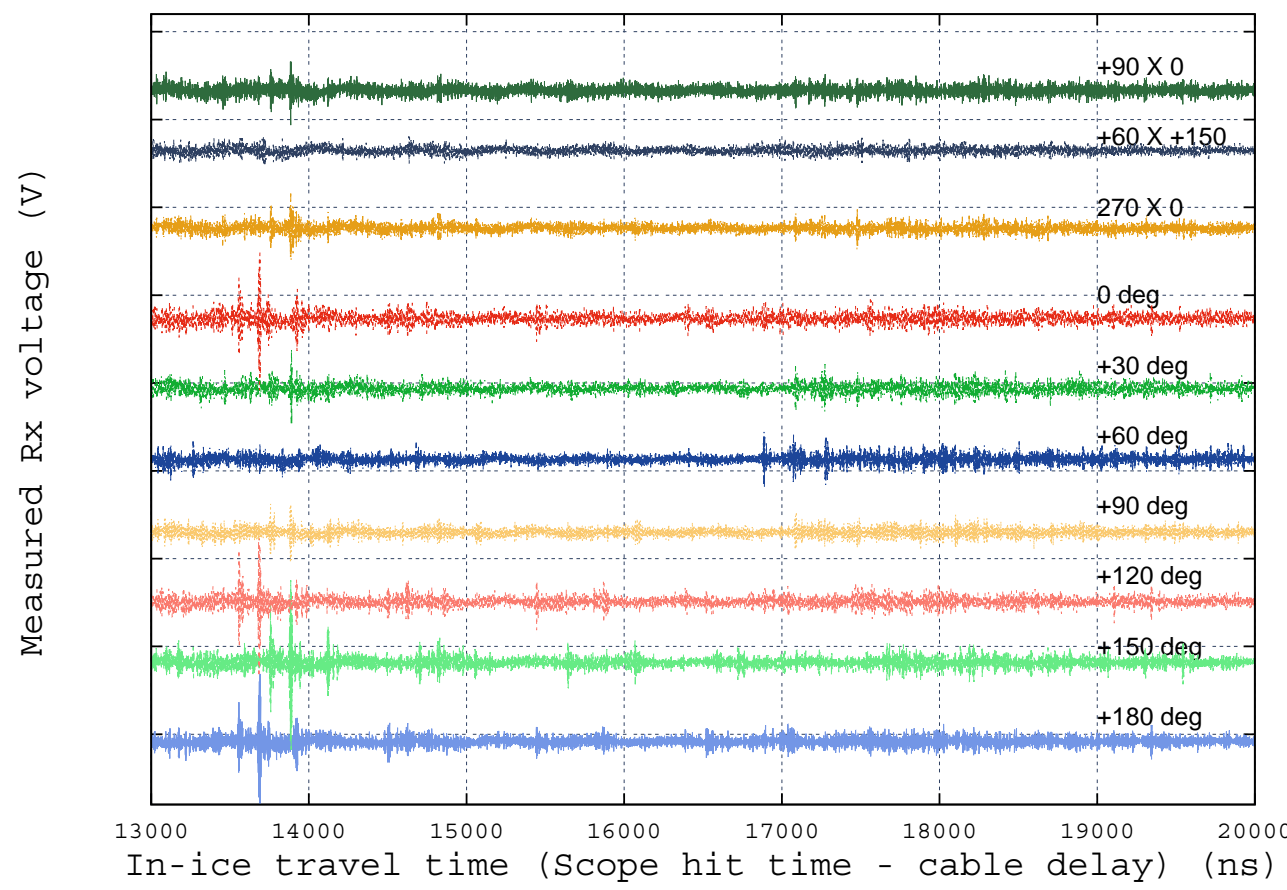

Fig. 4. Ensemble of echo amplitudes observed as a function of azimuthal orientation, for both co-polarized and cross-polarized broadcast signals, for echo times between $13 \mu \mathrm{s}$ and $20 \mu \mathrm{s}$. A DC offset has been added vertically to successive voltage traces for visual clarity; alternate waveforms are similarly also offset by $\pm 100 \mathrm{~ns}$ along horizontal. Each division vertically corresponds to $1 \mathrm{mV}$.

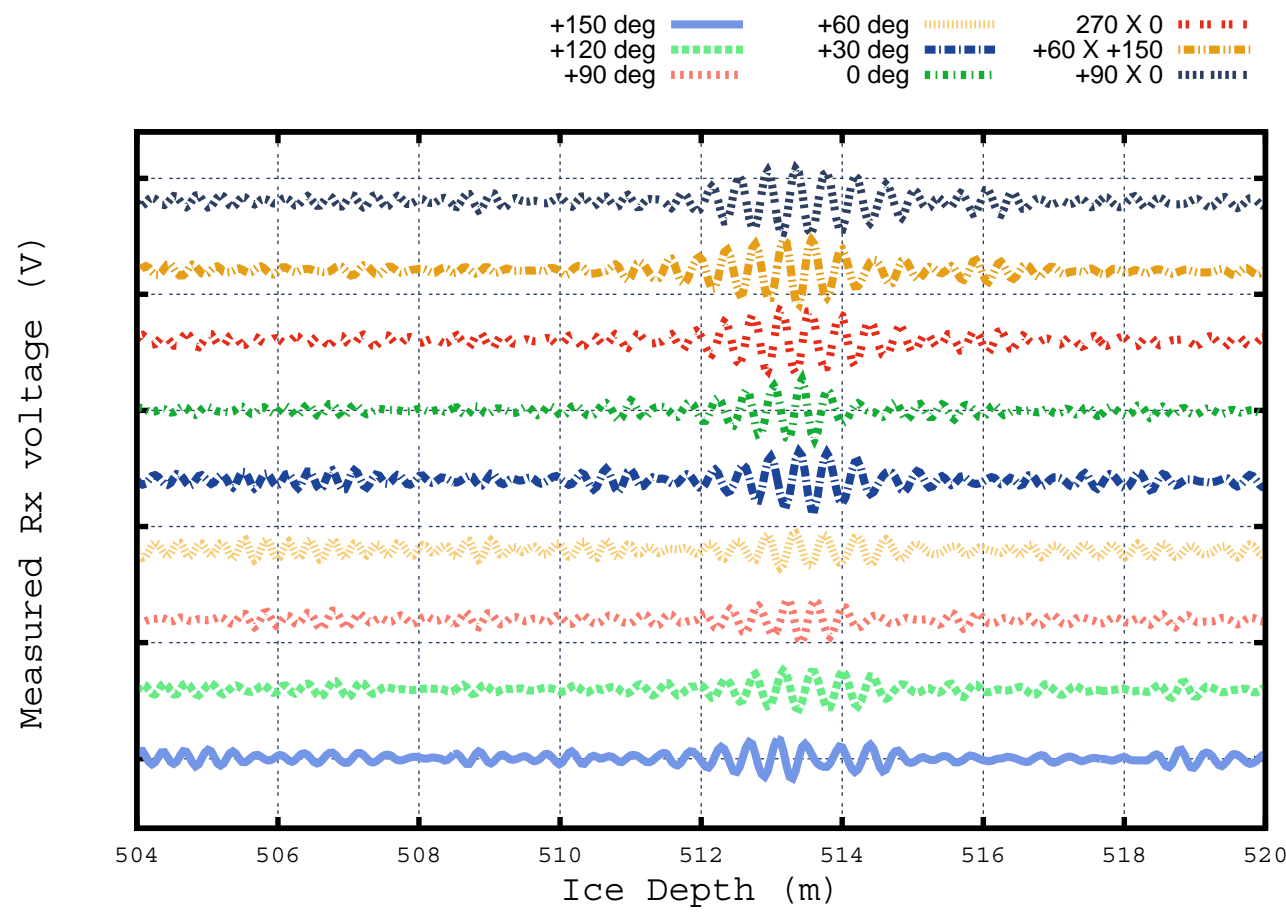

Fig. 5. Zoom of interval around $6 \mu \mathrm{s}$, converted to depth. Vertical divisions correspond to $10 \mathrm{mV}$. No horizontal offset has been applied to successive waveforms. Each 2 meter division horizontally corresponds to approximately $20 \mathrm{~ns}$. 


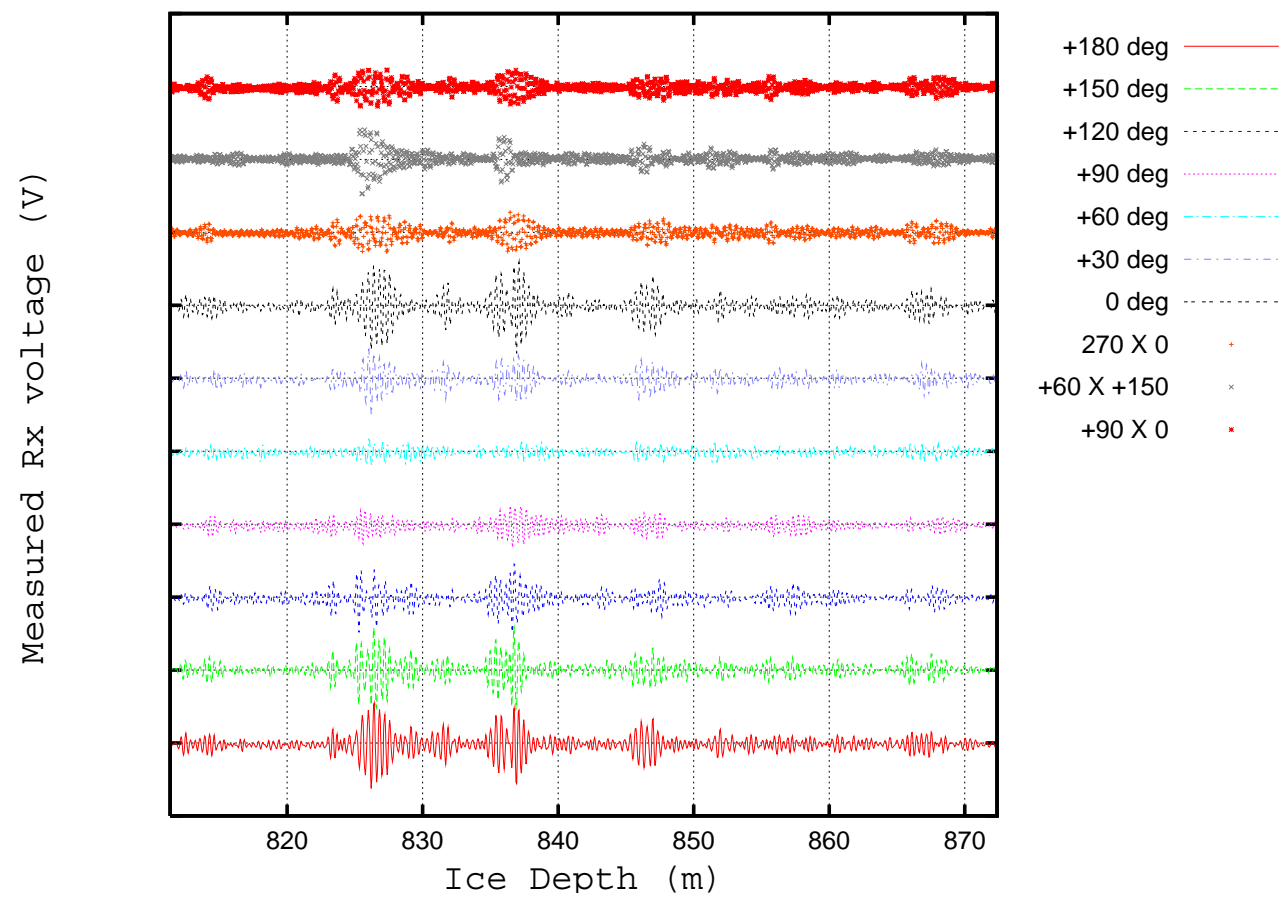

Fig. 6. Zoom of interval around $9.6 \mu$ s, converted to depth. Vertical divisions correspond to $5 \mathrm{mV}$. No horizontal offset has been applied to successive waveforms. Each 10 meter division horizontally corresponds to approximately $0.1 \mu$.

polarization and will not lead to more power observed in the cross-polarization orientation. Although we cannot exclude the possibility that this is indicative of the magnitude of the inherent voltage measurement errors, the fact that this is observed for all three cross-polarization orientations leads us to speculate on possible reasons.

The simplest explanation, of course, is that, for the case where the transmitted polarization is not coincident with either the extraordinary or ordinary axes, birefringence results in an apparent horizontal rotation of the net electric field vector. However, this model also requires a difference in the return times observed for polarizations corresponding to the birefringent basis directions. Assuming a "minimal" model of propagation at $45^{\circ}$ relative to the birefringent basis axes, phase differences of $\pm \pi / 8$ would result in both a longer signal observed in the cross-polarization orientation, as well as a time domain difference for co-polarization broadcasts along the birefringent basis directions. The required time difference for a quarter-wave rotation, assuming a typical in-ice period of $4 \mathrm{~ns}$ (i.e., frequency component of $250 \mathrm{MHz}$ ), is of order $1 \mathrm{~ns}$, at the limit of our timing resolution.

This explanation of our data is disfavored for two reasons: (a) the interference effect mentioned above will depend on frequency and will not always have the same sign, and (b) the two cross-polarization orientations for which data were taken are shifted azimuthally by $30^{\circ}$ relative to each other, so the fact that we observe "excessive" cross-polarization power in both orientations seems improbable.
A previously-undetected circular-polarizing capacity in ice could contribute to the cross-polarized signal amplitude. Such an amplitude variation might also be caused by, e.g., a conductive layer with a preferred alignment that results in a "grating-like" behavior. Such a grating would produce crosspolarized reflected power $\propto \sin \theta \cos \theta$, where $\sin \theta$ is the projection of the transmitter axis onto the grating axis, and $\cos \theta$ is the projection of the receiver axis onto the grating axis. That power would be greater than the co-polarized power for some orientations $\left(\sin \theta \cos \theta>\cos ^{2} \theta\right.$ for $\pi / 2>\theta>\pi / 4$, for instance).

It is also possible that the larger peak amplitudes are due to enhanced coherence of the frequency components which comprise our time domain signals, if the reflecting region is extended. In the simplest model, a uniform, flat reflecting layer due to simple density contrast is expected to produce an azimuthally uniform return. However, a sloped reflecting layer can produce an azimuthal variation in echo strength. The Fresnel zone has a radial extent $R$ roughly given by $R \sim \sqrt{2 \lambda d_{\mathrm{Tx}} d_{\mathrm{Rx}} /\left(d_{\mathrm{Tx}}+d_{\mathrm{Rx}}\right)} / 2$; using $\lambda \sim 1 \mathrm{~m}$ and $d_{\mathrm{Tx}} \sim d_{\mathrm{Rx}} \sim 1000 \mathrm{~m}$, we find $R \sim 30 \mathrm{~m}$. Nanosecond scale interference effects (the maximum allowed by Fig. 3) would therefore imply sloping of a reflecting layer by approximately $20 \mathrm{~cm} / 30 \mathrm{~m}$, or less than a degree. Such a gradual slope is not excluded by extant data. In principle, a lateral variation in the wavespeed over a Fresnel zone could also result in such interference. Such a variation might ostensibly 


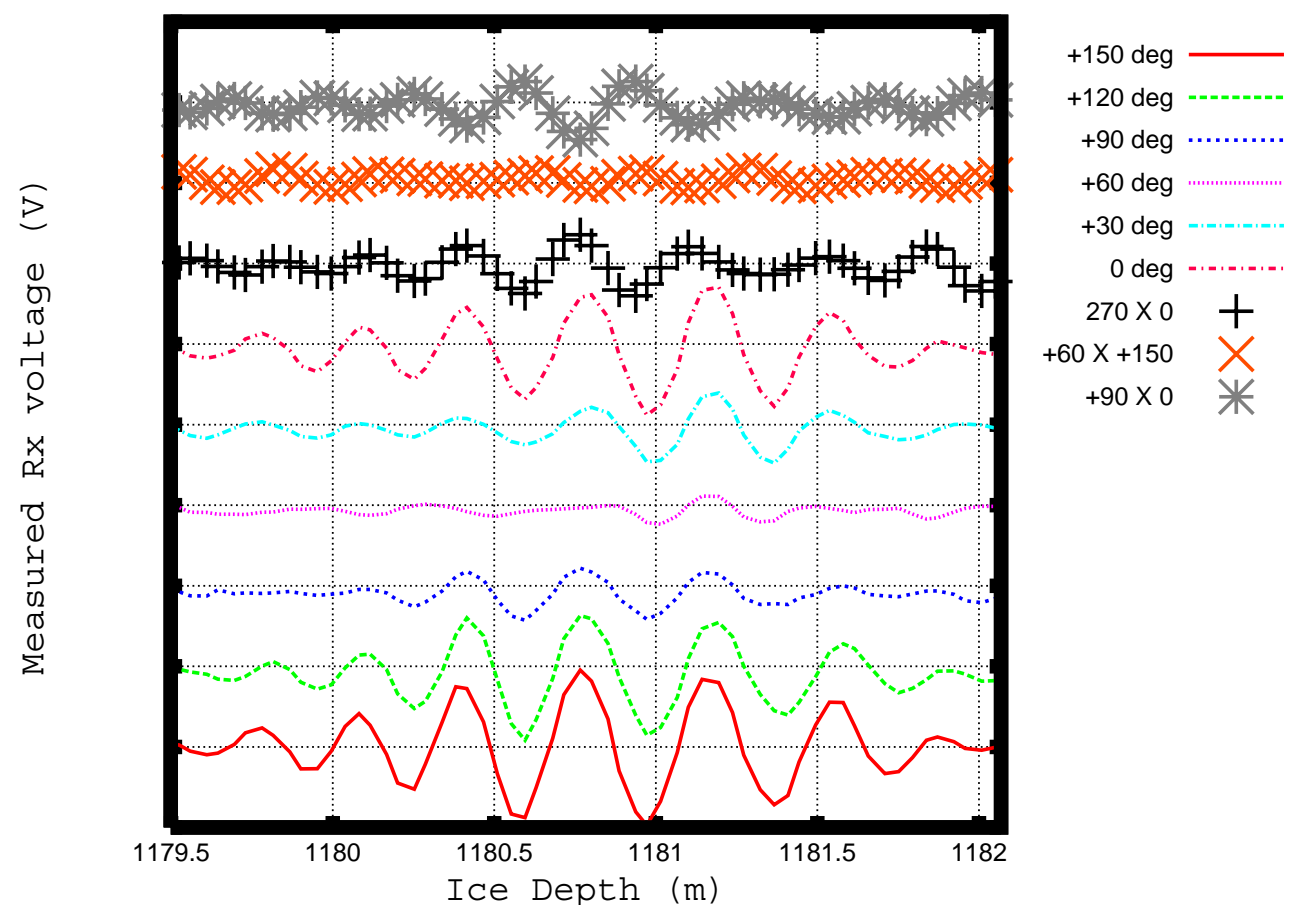

Fig. 7. Zoom of interval around $13.9 \mu$ s. Vertical divisions correspond to $1 \mathrm{mV}$. No horizontal offset has been applied to successive waveforms. Each 0.5 meter division horizontally corresponds to approximately $5 \mathrm{~ns}$.

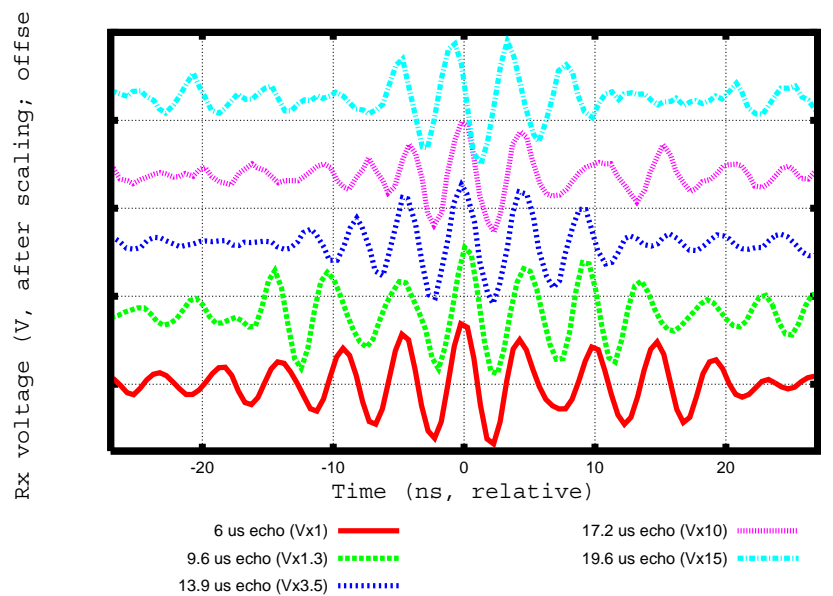

Fig. 8. Overlay of waveforms observed for various echoes. Amplitudes have been multiplied by indicated scale factors (in parentheses) to facilitate visual comparison.

be caused by slight variations in the overpressure above the layer.

The possibility that the cross-polarized power might be enhanced by Faraday rotation in ice has been considered, but is disfavored by laboratory results (Besson et al., 2009).

We note that in general, the "continuum" received power, away from evident peaks, in the cross-polarization orientation is not larger than the co-polarization power (presumably

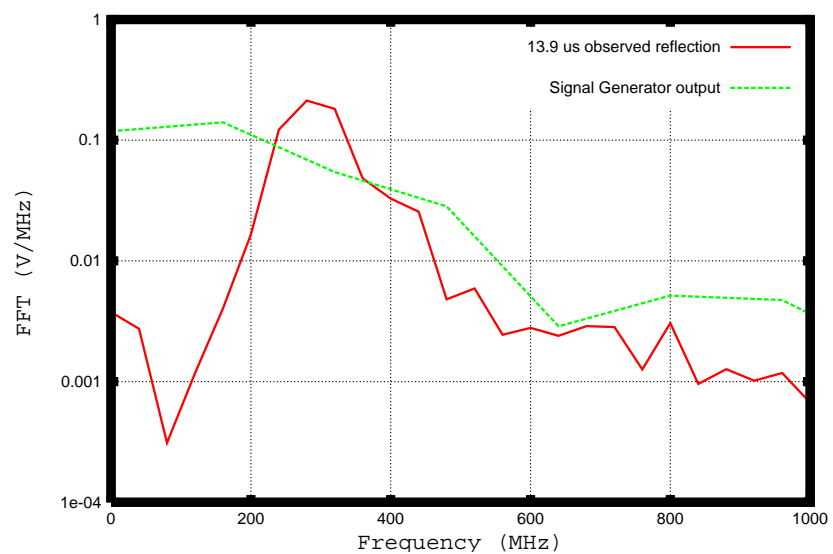

Fig. 9. Power spectrum comparison of direct signal generator output with observed reflection at $13.9 \mu$ s.

related to the geometric characteristics of volumetric scattering in the bulk ice, and indicating that the observed "large" cross-polarized power is intrinsic to layer scattering). This raises the possibility that the observed effects are a simple consequence of interference between planar layer reflection and volumetric Rayleigh scattering, and/or other multi-path effects. This is perhaps the most plausible explanation of our observations.

The ideal data sample to investigate this effect would include a full data sample, consisting of 8 azimuthal transmitter 


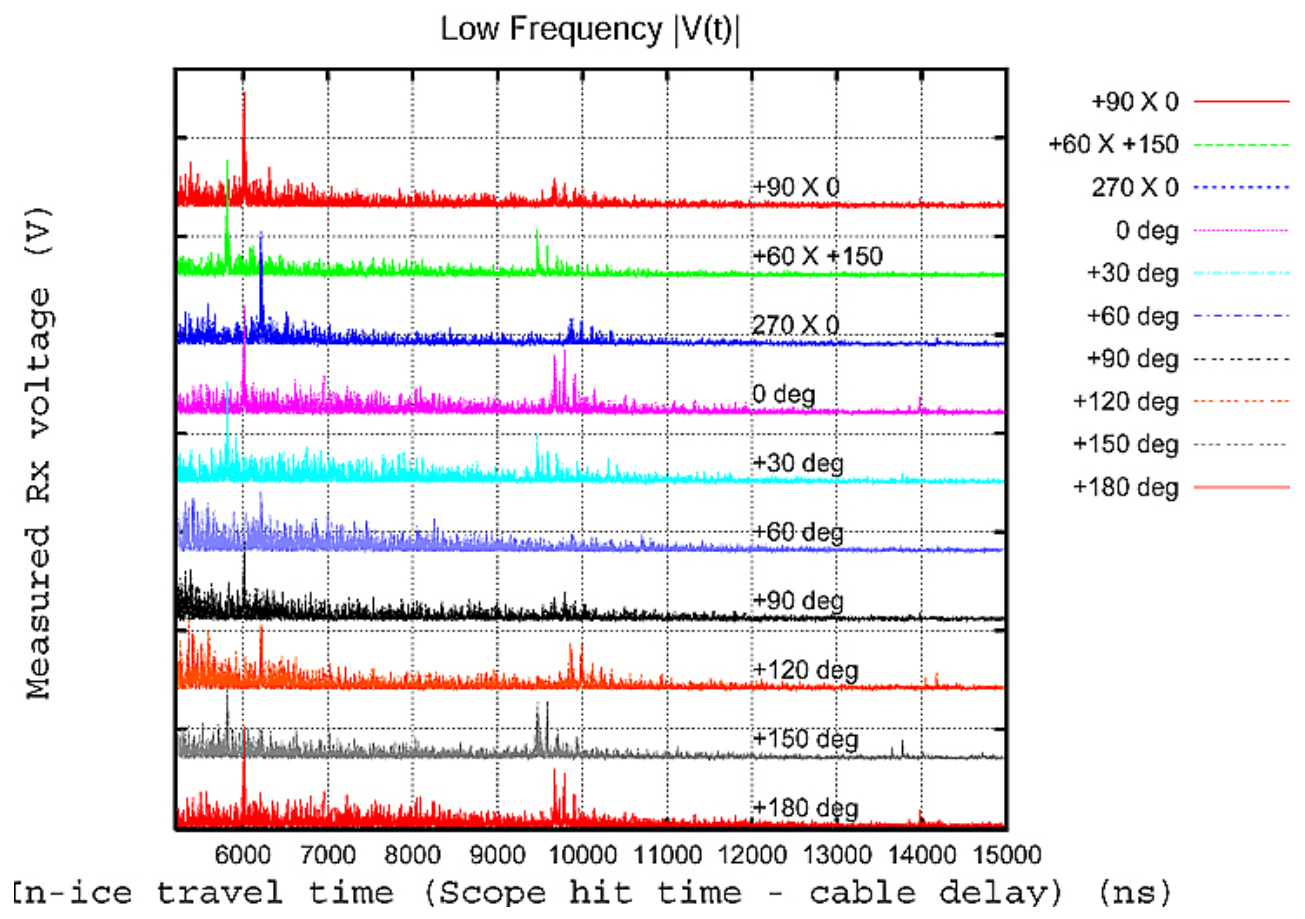

Fig. 10. Voltage magnitude after applying a $500 \mathrm{MHz}$ low-pass filter to the data shown in Fig. 3. Vertical divisions correspond to $5 \mathrm{mV}$.

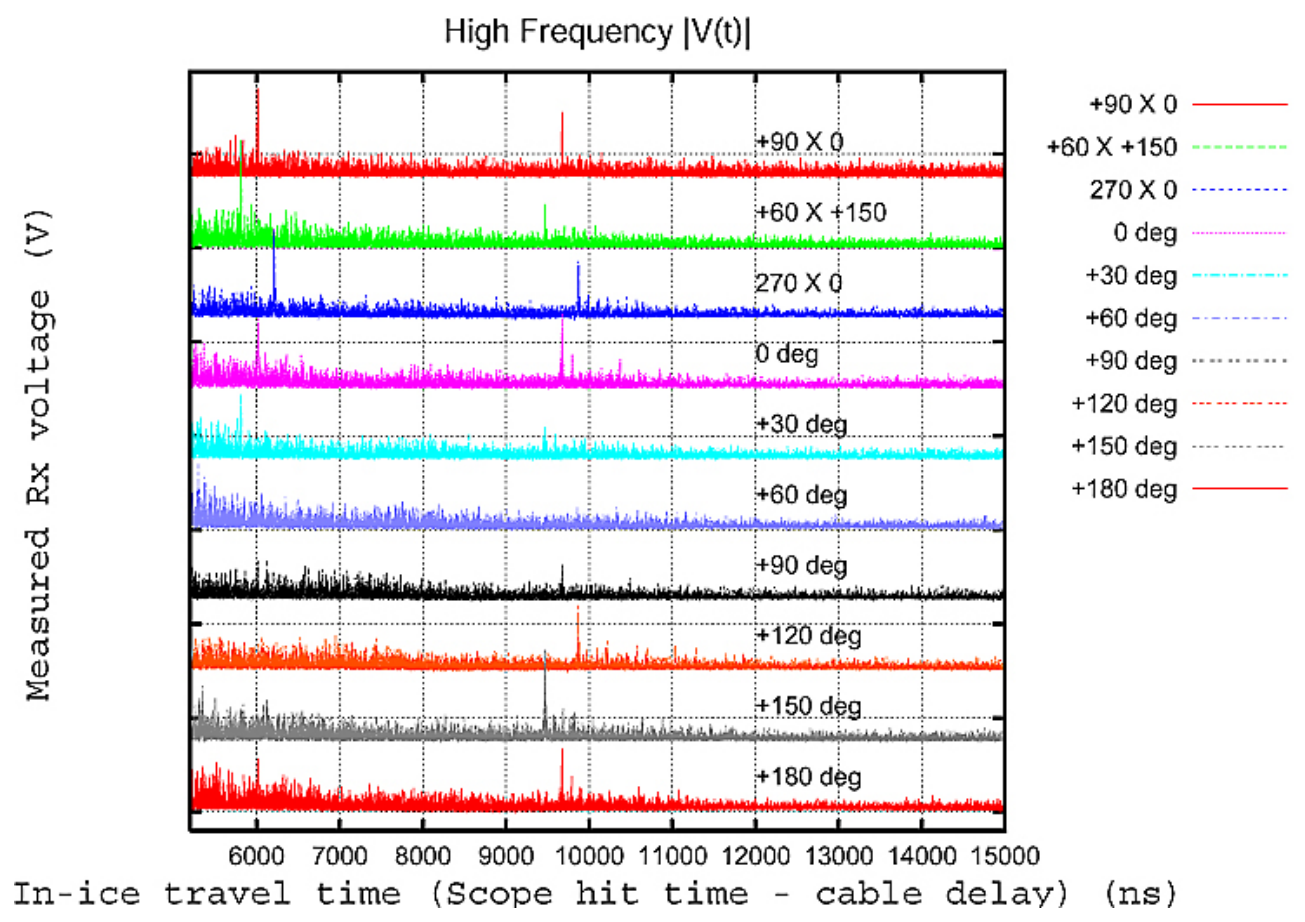

Fig. 11. Voltage magnitude after applying a $500 \mathrm{MHz}$ high-pass filter to the data shown in Fig. 3. Vertical divisions correspond to $0.2 \mathrm{mV}$.

polarizations, at $22.5^{\circ}$ intervals and with data taken at each of 8 azimuthal receiver polarizations, for a total of 64 data samples. This would ideally be complemented by data taken using "tone" signals to more cleanly observe slight birefringent effects based on phase differences. A frequency-banded data sample planned for the 2013-2014 austral season, with a higher power transmitter than those employed for these measurements, is currently being investigated to determine whether this effect is real or simply indicative of the magnitude of absolute systematic errors in our measurements. 


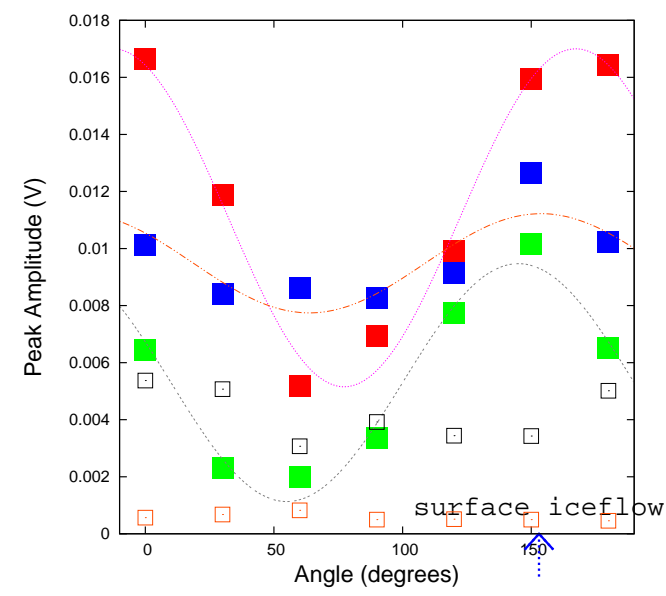

Fig. 12. Data of previous table presented in graphical form, with sinusoidal fits overlaid. Blue arrow indicates azimuth corresponding to local horizontal ice flow direction.

\subsection{Time domain characteristics of observed returns}

We have investigated the temporal and frequency characteristics of our observed echoes. Earlier East Antarctica studies concluded that "anisotropic scattering", occurring at depths where the alignment of the ice crystal fabric changes directions, is responsible for many of the echoes observed at intermediate depths. Such scattering of the COF type should be independent of frequency over the interval $60-179 \mathrm{MHz}$ (Matsuoka et al., 2003). COF scattering can thus be distinguished from acid layer scattering, which features amplitude believed to vary inversely as frequency, but not from density layer scattering.

Figure 8 compares zooms of the time domain waveforms of the reflections observed at 6, 9.6, 13.9, 17 and $19 \mu \mathrm{s}$. We observe that the shallower time-domain returns are, if anything, longer in duration than the deeper returns. Given the falling power spectrum of the transmitted signal (the Fourier transform of Fig. 2), this indicates higher fractional signal content at lower frequencies. Overall, the similarity of the waveforms suggests that the same scattering mechanism may be responsible for all observed reflections. A direct comparison of the power spectrum for one of reflections $(13.9 \mu \mathrm{s}$, shown in Fig. 9) with the signal generator output shows a diminishing ratio of reflected power relative to signal output power, favoring the $1 / f$-dependent conductivity-type scattering interpretation. The sharpness of the reflections, given the fact that $\mathrm{COF}$ re-alignment is expected to occur over several meters, additionally suggests that all our observed reflections are due to conduction layers.

We note that the depths for the two sharpes, initial echoes recorded around 9.6 microseconds in Fig. 6, and separated by an estimated $10 \mathrm{~m}$ vertically, correspond (within roughly $10 \mathrm{~m}$ ) to the depths of two nearly adjacent ash layers directly measured by an optical hole logging system, originally de-

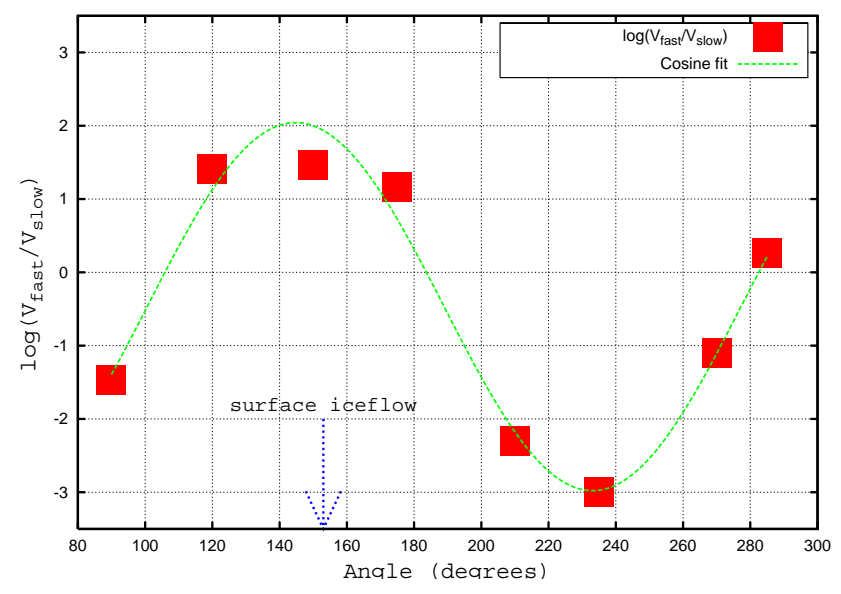

Fig. 13. Ratio of amplitudes for "fast" vs. "slow" reflection signals, as a function of azimuthal orientation of co-polarized surface horns. Blue arrow indicates azimuth corresponding to local horizontal ice flow direction.

signed to measure optical impurities in holes drilled for the IceCube experiment (Abassi et al., 2012). This lends strong support to the interpretation that our reflection layers are due to conductivity scattering rather than crystal re-orientation.

One may ask if our observations of the time-domain reflection characteristics are a simple consequence of ice attenuation effects, which are expected to increase with frequency over this frequency range (Fujita et al., 1996). The waveform shapes, qualitatively, disfavor a model wherein ice attenuation increases with frequency, as this would tend to reduce the sharpness of the later rather than earlier returns.

One may also ask whether the observed signal lengths might simply result from multiple layers giving reflections on a time scale shorter than the observed $20 \mathrm{~ns}$ signal durations. Assuming a $10 \mathrm{~cm} \mathrm{yr}^{-1}$ accumulation rate at South Pole, such a model would require not only significant layer depositions within 20 years of each other, but would also tend to produce asymmetric observed signals. In general, the symmetry of our observed time domain signals appears to argue against such a model.

\subsubsection{Frequency domain characteristics}

Figures 10 and 11 show the time domain voltage magnitude, after applying software filters of $f<500 \mathrm{MHz}$ and $f>500 \mathrm{MHz}$, respectively, and transforming back into the time domain. (As $600 \mathrm{MHz}$ represents the center frequency of our bandpass, the selection of $500 \mathrm{MHz}$ results in intervals with unequal bandwidths but - since the antenna voltage response varies as the inverse of frequency - more comparable signal-to-noise.) We observe comparable fractional highfrequency power for the $9.6 \mu \mathrm{s}$ return compared to the $6 \mu \mathrm{s}$ reflection, consistent with the qualitative conclusion drawn from the time domain waveforms shown in Fig. 8. 
Table 2. Inter-layer attenuation lengths, calculated from amplitudes measured for returns and assuming uniform reflectivity of all layers, as discussed in text. First column indicates first reflecting layer; successive columns indicate second reflecting layer used to calculate attenuation length via Friis equation. Estimated errors are of the order $30 \%$.

\begin{tabular}{lccc}
\hline & $13.9 \mu \mathrm{s}$ & $17.2 \mu \mathrm{s}$ & $19.6 \mu \mathrm{s}$ \\
\hline $6 \mu \mathrm{s}$ & $3348 \mathrm{~m}$ & $1521 \mathrm{~m}$ & $1514 \mathrm{~m}$ \\
$9.6 \mu \mathrm{s}$ & $1170 \mathrm{~m}$ & $867 \mathrm{~m}$ & $964 \mathrm{~m}$ \\
$13.9 \mu \mathrm{s}$ & & $643 \mathrm{~m}$ & $849 \mathrm{~m}$ \\
\hline
\end{tabular}

\subsection{Attenuation length dependence on depth and temperature}

The observed echo amplitudes shown in Fig. 8 are largely determined by three factors: the intrinsic reflectivity of each layer, the diminution of signal strength with distance, and attenuation of the signal due to continuous ice absorption.

We can test the assumption that the scattering mechanisms for all reflection layers are the same by investigating whether the amplitude dependence of the observed reflections is consistent with the known warming of ice with depth, and the known reduction in ice attenuation length at radio frequencies with increasing temperature.

We determine a "local" amplitude attenuation length $L_{\text {atten }}$ between the first three and last three layers, as shown in Table 2, by assuming specular reflection and directly applying the Friis equation (Balanis, 1997): $V_{\mathrm{Rx}} / V_{\mathrm{Tx}}=$

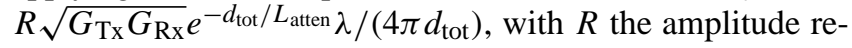
flectivity of each layer, $V_{\mathrm{Rx}}$ and $V_{\mathrm{Tx}}$ the measured received signal voltage and transmitted signal voltage, respectively, $d_{\text {tot }}$ the total round-trip echo path, $\lambda$ the broadcast wavelength, and $G$ the antenna gain of transmitter and receiver antenna, taken to be $12 \mathrm{dBi}$ given the expected in-ice focusing.

Our estimates in Table 2 show a general trend of shorter attenuation lengths closer to the bed, consistent with the expected warming of the ice sheet from below and the corresponding reduction in attenuation length with increasing temperature. Our measurements therefore support the notion that the intrinsic reflectivities of the reflection layers are similar. We note that the temperature dependence of conductivity scattering would lead to a reduction in reflection coefficient with depth and could therefore also account for some of the variation observed; in Table 2 , we neglect the $6^{\circ} \mathrm{K}$ difference in layer temperature between the depth implied for the $13.9 \mu$ s reflection vs. the $19.6 \mu$ s reflection (roughly 1180 and $1650 \mathrm{~m}$, respectively), based on the model of Price et al. (Price et al., 2002).
Table 3. Peak voltages (in $\mathrm{mV}$ ) for indicated reflections as a function of angle. Asterisks indicate very low observed voltage signal : noise.

\begin{tabular}{lccccc}
\hline Orientation & $6 \mu \mathrm{s}$ & $9.6 \mu \mathrm{s}$ & $13.9 \mu \mathrm{s}$ & $17 \mu \mathrm{s}$ & $19 \mu \mathrm{s}$ \\
\hline$+270 \times+0 \mathrm{deg}$ & 28.5 & 5.6 & 2.0 & 1.2 & $0.7^{*}$ \\
$+90 \times+0 \mathrm{deg}$ & 31.4 & 5.7 & 1.9 & $0.96^{*}$ & 0.8 \\
$+60 \times+150 \mathrm{deg}$ & 30.5 & 12.8 & $0.9^{*}$ & 0.7 & $0.6^{*}$ \\
$+180 \mathrm{deg}$ & 27.4 & 16.4 & 3.3 & $0.9^{*}$ & 1.0 \\
$+150 \mathrm{deg}$ & 17.9 & 15.9 & 5.1 & $1.0^{*}$ & 1.3 \\
$+120 \mathrm{deg}$ & 18.8 & 9.9 & 3.8 & $1.0^{*}$ & 0.9 \\
$+90 \mathrm{deg}$ & 21.1 & 6.9 & 1.7 & $1.0^{*}$ & $0.8^{*}$ \\
$+60 \mathrm{deg}$ & 14.9 & 5.2 & $1.0^{*}$ & $1.6^{*}$ & $0.8^{*}$ \\
$+30 \mathrm{deg}$ & 29.6 & 11.8 & $1.2^{*}$ & 1.3 & 0.8 \\
$0 \mathrm{deg}$ & 25.4 & 16.6 & 3.0 & 1.1 & 1.0 \\
\hline
\end{tabular}

\subsection{Variation in return amplitude with azimuth}

A comparison of the maximum amplitudes observed for the five primary reflections is presented in Table 3; asterisks indicate that the reflection at that depth and orientation angle was largely embedded in noise. Figure 12 displays the peak amplitude voltages vs. co-polarization orientation angle. For three of these layers, we observe an apparent co-sinusoidal variation which is roughly in phase with the ice flow direction $\left(153^{\circ}\right.$, shown as the blue arrow in the figure); no obvious correlation is apparent for the $6 \mu$ s and $19 \mu$ s reflections.

Mechanisms responsible for observed azimuthal dependences in radio echo soundings were outlined nearly 40 years ago (Hargreaves, 1977, 1978). In our case, the azimuthal variation observed is otherwise unrelated to the azimuthal variation reported in East Antarctica (Fujita et al., 2006); in particular, those variations were attributed to interference between projections of the radio-frequency electric field vector propagating along two orthogonal axes. In our case, there is no obvious operative interference mechanism. I.e., the synchronicity (to within 1 nanosecond) of the reflections, at all polarizations, rules out the possibility that the observed azimuthal amplitude variation for these reflections in the upper half of the ice sheet results from birefringence-induced interference effects.

A previous paper (Kravchenko et al., 2010) investigated the basal echo times at South Pole as a function of polarization. That study demonstrated that reflections through the full ice sheet, off the bedrock, do exhibit the azimuthal dependence of echo times and voltages characteristic of birefringence. Specifically, for polarizations aligned parallel to the putative ordinary axis, only one return is observed, with a voltage $V_{\text {fast }}^{\max }$ and a negative echo time offset relative to that observed for polarizations aligned with the putative extraordinary axis, presumably rotated by $\pi / 2$ radians. If we designate the voltage observed for alignments parallel to the ordinary axis as $V_{\text {fast }}^{\max }$, then, for polarizations at $\pi / 4$ radians relative to each axis, two returns should be observed, 
each with amplitude $1 / \sqrt{2}$ as large as the co-aligned case; i.e., $V_{\text {fast }}(\pi / 4) / V_{\text {slow }}(\pi / 4)=1$. Additionally, for this case, $V_{\text {fast }} / V_{\text {slow }}=V_{\text {fast }}^{\max } / \sqrt{2}$. $V_{\text {fast }} / V_{\text {slow }}$ (Fig. 13, Kravchenko et al., 2010) shows measurements consistent with these expectations, and thus a correlation consistent with ice flow direction defining the extraordinary axis in the lower half of the ice sheet.

\section{Conclusions}

We have observed azimuthal correlations of echo returns with ice flow direction at South Pole, which we summarize as follows:

1. Although previous probes of the ice sheet in East Antarctica (Fujita et al., 2006) were reported as birefringence in the upper half of the ice sheet, we observe birefringent effects predominantly in the lower half of the ice sheet. Both experiments observe correlations of birefringence with the ice flow direction.

2. In the "standard" picture, if the c-axis has an isotropic distribution around the vertical, and the wavespeed asymmetry is different only for propagation parallel (i.e., along z) vs. perpendicular (horizontal) to the crystal stacking axis (i.e., $\hat{c}$ ), then the wavespeed is uniform for all directions in the horizontal plane and there is no expected birefringence as a function of azimuthal orientation. However, our results imply an asymmetry for azimuthal propagation along vs. perpendicular to the ice flow direction, in contrast to measurements for single crystals, which would have implied azimuthal symmetry. Our results are consistent with elliptical ice fabric in the shallowest half of the ice sheet and a vertical girdle average orientation at depths greater than $\sim 1600 \mathrm{~m}$, although ice core analysis indicates that the ice should be increasingly uniaxial at these depths.

Two additional inputs could significantly clarify the association between radio echo sounding measurements and ice structure and composition: (1) an ice core taken at South Pole, preferably retaining the azimuthal information of the extracted core itself (perhaps unlikely given the operational challenge this presents), or (2) ns-duration probes of the Antarctic ice sheet at a location where a core has already been taken (e.g., Vostok, or WAIS). Such data will hopefully be available in the near future.
Acknowledgements. The authors particularly thank Chris Allen and John Paden (University of Kansas), John Ralston (University of Kansas), Rebecca Boon (Pennsylvania State University), Joe MacGregor (University of Texas), and Kenny Matsuoka (Norwegian Polar Institute) for very helpful discussions, as well as our colleagues on the RICE and ANITA experiments. We also thank Andy Bricker of Lawrence High School (Lawrence, KS) for his assistance working with Lawrence High School students who performed essential antenna calibrations. This work was supported by the National Science Foundation's Office of Polar Programs (grant OPP-0826747) and QuarkNet programs. Any opinions, findings, and conclusions or recommendations expressed in this material are those of the author(s) and do not necessarily reflect the views of the National Science Foundation.

Edited by: J. L. Bamber

\section{References}

Abassi, R. and 245 others: South Pole glacial climate record from borehole laser particulate stratigraphy, submitted to J. Glaciol, 2013.

Allison, P. and 47 others: Design and initial performance of the Askaryan Radio Array prototype EeV neutrino detector at the South Pole, Astropart. Phys., 35, 457-477, 2011.

Azuma, N. and Higashi, A.: Formation processes of ice fabric pattern in ice sheets, Ann. Glaciol. 6, 130-134, 1985.

Balanis C.: Antenna Theory, Analysis and Design, J. Wiley and Sons, New York City, NY, 1997.

Barwick, S., Besson, D., Gorham, P., and Saltzberg, D.: South Polar in situ Radio Frequency Ice Attenuation, J. Glaciol, 51, 231-238, 2005.

Besson, D. Z. and 42 others: In situ radioglaciological measurements near Taylor Dome, Antarctica and implications for UHE neutrino astronomy, Astropart. Phys., 29, 130-157, 2008.

Besson, D., Keast, R., and Velasco, R.: In situ and laboratory studies of radiofrequency propagation through ice and implications for siting a large-scale Antarctic neutrino detector, Astropart. Phys., 31, 348-358, 2009.

Doake, C. S. M.: Polarization of Radio Waves Propagated through George VI Ice Shelf, British Antarctic Survey Bulletin, 1-6, 1982.

Doake, C., Corr, H., and Jenkins, A.: Polarization of radio waves transmitted through, Antarctic ice shelves Ann. Glaciol., 34, 165-170, 2002.

Doake, C., Corr, H., Jenkins, A., Nichols, K., and Stewart, C.: Applications of SAR Polarimetry and Polarimetric Interferometry, Euro. Space Agency, 529, 313-320, 2003.

Dowdeswell, J. A. and Evans, S.: Investigations of the form and flow of ice sheets and glaciers using radio-echo sounding, Rep. Prog. Phys., 67, 1821-1863, 2004.

Drews, R., Eisen, O., Weikusat, I., Kipfstuhl, S., Lambrecht, A., Steinhage, D., Wilhelms, F., and Miller, H.: Layer disturbances and the radio-echo free zone in ice sheets, The Cryosphere, 3, 195-203, doi:10.5194/tc-3-195-2009, 2009.

Eisen, O., Hamann, I., Kipfstuhl, S., Steinhage, D., and Wilhelms, F.: Direct evidence for continuous radar reflector originating 
from changes in crystal-orientation fabric, The Cryosphere, 1, 1-10, doi:10.5194/tc-1-1-2007, 2007.

Fujita, S. and Mae, S.: Relation between ice sheet internal radioecho reflections at Mizuho Station, Antarctica, Ann. Glaciol. 17, 269-275, 1993.

Fujita, S., Matsuoka, T., Morishima, S., and Mae, S.: The measurement on the dielectric properties of ice at HF, VHF and microwave frequencies, Geosci. Remote Sens. Symp., IGARSS '93, Abstract \#48, 1258-1260, 1996.

Fujita, S., Maeno, H., Uratsuka, S., Furukawa, T., Mae, S., Fujii, Y., and Watanabe, O.: Nature of radio echo layering in the Antarctic ice sheet detected by a two-frequency experiment, J. Geophys. Res., 104, 13, 1999.

Fujita, S., Matsuoka, T., Ishida, T., Matsuoka, K., and Mae, S.: A summary of the complex dielectric permittivity of ice in the megahertz range and its application for radar sounding of polar ice sheets, Physics of Ice Core Records, edited by: Hondoh, T., Hokkaido University Press, Sapporo, 185-212, 2000.

Fujita, S., Matsuoka, K., Maeno, H., and Furukawa, T.: Scattering of VHF radio waves from within an ice sheet containing the vertical-girdle-type ice fabric and anisotropic reflection boundaries, Ann. Glaciol. 37, 305-316, 2003.

Fujita, S., Maeno, H., and Matsuoka, K.: Radio-wave depolarization and scattering within ice sheets: a matrix-based model to link radar and ice-core measurements and its application, J. Glaciol, 52, 407-424, 2006.

Hargreaves, N. D.: The polarization of radio signals in the radio echo sounding of ice sheets, J. Phys. D, Appl. Phys, 10, 12851304, 1977.

Hargreaves, N. D.: The radio frequency birefringence of polar ice, J. Glaciol, 21, 301-313, 1978.

Jacka, T. H. and Jun, Li: The steady-state crystal size of deforming ice, Ann. Glaciol. 20.1, 13-18, 1994.

Kamb, W. Barclay, Theory of preferred crystal orientation developed by crystallization under stress, J. Geol., 153-170, 1959.

Kravchenko, I., Besson, D., and Meyers, J.: In situ index of refraction measurements of the South Polar firn with the RICE detector, J. Glaciol, 50, 522-532, 2004.

Kravchenko, I., Besson, D., Ramos, A., and Remmers, J.: Radio frequency birefringence in South Polar ice and implications for neutrino reconstruction, Astropart. Phys. 34, 10, 755-768; and: http://xxx.lanl.gov/pdf/1005.4589, 2010.
Matsuoka, K., Furukawa, T., Fujita, S., Maeno, N., Uratsuka, S., Naruse, R., and Watanabe, O.: Crystal orientation fabrics within the Antarctic ice sheet revealed by a multipolarization plane and dual-frequency radar survey, J. Geophys. Res., 108, B10, 2003.

Matsuoka, K., Uratsuka, S., Fujita, S., and Nishio, F.: Ice-flowinduced scattering zone within the Antarctic ice sheet revealed by high-frequency airborne radar, J. Glaciol, 50, 170, 382-388, 2004.

Matsuoka, K., Wilen, L., Hurley, S. P., and Raymond, C. F.: Effects of Birefringence Within Ice Sheets on Obliquely Propagating Radio Waves, IEEE T. Geosci. Remote Sens., 47, 1429-1443, 2009.

Matsuoka, T., Fujita, S., Morishima, S., and Mae, S.: Precise measurement of dielectric anisotropy in ice Ih at $39 \mathrm{GHz}$, J. Appl. Phys., 81, 2344-2348, 1997.

Matsuoka, T., Mae, S., Fukazawa, H., Fujita, S., and Watanabe, O.: Microwave dielectric properties of the ice core from Dome Fuji, Antarctica, Geophys. Res. Lett., 25, 1573, 1576, 1998.

Matsuoka, K., Power, D., Fujita, S., and Raymond, C. F.: Rapid development of anisotropic ice-crystal-alignment fabrics inferred from englacial radar polarimetry, central West Antarctica, J. Geophys. Res., 117, F03029, 2012.

Paren, J.: Reflection coefficient at a dielectric interface, J. Glaciol., 27, 203-204, 1981.

Price, P.B. and 9 others, Temperature profile for glacial ice at the South Pole: Implications for life in a nearby subglacial lake, Proc. Nat. Acad. Sci., 99, 7844-7877, 2002.

Schmid, S. M. and Casey, M. : Complete fabric analysis of some commonly observed quartz c-axis patterns, Geophys. Monogr., 36, 263-286, 1986.

Siegert, M. J. and Kwok, R.: Ice-sheet radar layering and the development of preferred crystal orientation fabrics between Lake Vostok and Ridge B, central East Antarctica, Earth Planet. Sci. Lett., 179, 227-235, 2000.

Van der Veen, C. J. and Whillans, I. M.: Development of fabric in ice, Cold regions science and technology 22.2, 171-195, 1994.

Wang, Y., Kipfstuhl, J., Miller, H., Thorsteinsson, T., Dahl-Jensen, D., and Shoji, H.: A vertical girdle fabric in the NorthGRIP deep ice core, North Greenland, Ann. Glaciol., 35, 515-520, 2002.

Woodruff, A. H. and Doake, C. S. M.: Depolarization of radio waves can distinguish between floating and grounded ice sheets, J. Glaciol., 23, 223-232, 1979. 